\title{
Profiling of matrix metalloproteinases and tissue inhibitors of metalloproteinases proteins in bladder urothelial carcinoma
}

\author{
CHENG-KENG CHUANG ${ }^{1}$, SEE-TONG PANG ${ }^{1}$, TAI-JUNG CHUANG ${ }^{1}$ and SHUEN-KUEI LIAO ${ }^{2}$ \\ ${ }^{1}$ Department of Surgery, Division of Uro-Oncology, Chang Gung Memorial Hospital; \\ ${ }^{2}$ Graduate Institute of Clinical Medical Science, Chang Gung University, Taoyuan, Taiwan, R.O.C.
}

Received June 26, 2009; Accepted May 6, 2010

DOI: 10.3892/ol_00000121

\begin{abstract}
We investigated the matrix metalloproteinases (MMPs) and tissue inhibitors of metalloproteinase (TIMPs) proteins in transitional cell carcinoma (TCC) cell lines and surgical specimens of the bladder neoplasm. The expression level was correlated to the degree of cellular differentiation and invasiveness of bladder cancer. Panels of six TCC cell lines with different degrees of differentiation were tested with monoclonal antibodies (mAbs) to MMP-1, MMP-2, MMP-3, MMP-9a, MMP-9b, TIMP-1 and TIMP-2 by immunocytochemistry. Gelatin zymography was also conducted on the cell lines for MMP-2 and -9. In addition, immunohistochemistry with the mAbs to MMP and TIM molecules was performed on 30 TCC specimens. We found that TCC cell lines were stained positively for MMP-1 (6/6), weakly for MMP-9a (2/6), MMP9b (5/6) and TIMP-1 (3/6), and negatively for MMP-2 (3/6) and MMP-3 (3/6). Zymographic analysis of the cell lines showed a high level of MMP2 in the MGH-U4 cell line. In bladder cancer surgical specimens, all specimens were positive for MMP1 (30/30), 19 were positive for MMP-2 (63.3\%), 21 positive for MMP-9a (70\%) and 15 positive for MMP-9b (50\%). The expression of MMP-2 was found to be positively correlated with higher-grade tumors $(\mathrm{p}=0.036)$ and the expression of MMP-9a and $-9 b$ was found to be positively correlated with tumor stage $(\mathrm{p}=0.012$ and 0.023 , respectively). However, the expression of MMP-1, MMP-3, TIMP-1 and TIMP-2 was not correlated with either tumor staging or grading. In conclusion, the expression of MMP-2 and -9 was correlated with high-grade or high-stage bladder tumors, respectively. However, this correlation was not observed with TCC cell lines in which high- and low-grade tumors are included. Immunohistochemical results on tumor lesions may have more clinical relevance, since in a given tumor
\end{abstract}

Correspondence to: Dr Cheng-Keng Chuang, Department of Surgery, Division of Uro-Oncology, Chang Gung Memorial Hospital, Chang Gung University, 5 Fushing St., Kweishan, Taoyuan, 333 Taiwan, R.O.C. E-mail: chuang89@cgmh.org.tw

Keywords: bladder neoplasm, metastasis, matrix metalloproteinases, cell line, tissue inhibitors of metalloproteinases microenvironment the interaction among tumor cells in situ and tumor-associated cells, such as neutrophils, macrophages, lymphocytes and endothelial cells, as well as environmental factors (hypoxia and $\mathrm{pH}$ ), cytokines and growth factors released by these cells may be required for TCC to express selective MMPs and TIMPs. The selective expression of these molecules then regulates tumor progression.

\section{Introduction}

Metastasis is the leading cause of death in bladder urothelial carcinoma patients. Several events are required for metastasis to occur, including neovascularization, cell attachment, invasion and cell proliferation. Interactions of neoplastic cells with the extracellular matrix are critical steps in the growth and invasion of cancer. These interactions have been demonstrated in a wide range of human cancers, including urothelial cancers (1). Matrix metalloproteinases (MMPs), a family of $\mathrm{Zn}^{2+}$-dependent endogenous proteases, are able to degrade various components of extracellular matrix. In the extracellular domain, the activity of these proteases is tightly regulated by inhibitors known as tissue inhibitors of metalloproteinases (TIMPs). It has been postulated that TIMPs act as tumor suppressor genes due to their anti-metalloproteinase activity and their protective role on the extracellular matrix. The imbalance between MMPs and TIMPs may be an indicator for cancer prognosis. To investigate the role of MMPs and TIMPs in urinary bladder cancers, the expression of MMPs and TIMPs in bladder transitional cell carcinoma (TCC) cell lines, as well as surgical specimens was investigated in order to see whether these specimens were an indicator of cancer prognosis.

\section{Materials and methods}

Cell lines and surgical specimens. Six transitional cell carcinoma cell lines (MGH-U1, MGH-U1R, MGH-U3, MGH-U4, TCC8704 and TSGH8301) were used. The cell lines were main-tained in RPMI-1640 (Gibco) containing 10\% heatinactivated fetal bovine serum (FBS), $50 \mathrm{U} / \mathrm{ml}$ penicillin-G and $50 \mu \mathrm{g} / \mathrm{ml}$ streptomycin (Gibco), $2 \mathrm{mM}$ L-glutamine and 1 mM sodium pyruvate (Gibco). The four human bladder cancer cell lines, MGH-U1, -U1R, -U3 and -U4, were generous gifts from Dr C.W. Lin (Massachusetts General Hospital, Boston, 
Table I. Expression of MMPs and TIMPs in TCC cell lines determined by immunocytochemistry.

\begin{tabular}{lccccccccc}
\hline Cell lines & Stage $^{\mathrm{a}}$ & Grade & HLA-ABC & MMP1 & MMP2 & MMP3 & MMP9a & MMP9b & TIMP1 \\
\hline MGH-U1 & B & 3 & $2+$ & $3+$ & - & - & $1+$ & $1+$ \\
MGH-U1R & B & 3 & $2+$ & $3+$ & - & - & - & $1+$ & \pm \\
MGH-U3 & A & 1 & $3+$ & $2+$ & $2+$ & $2+$ & \pm & $1+$ & - \\
MGH-U4 & O & atypia & $3+$ & $3+$ & $3+$ & $2+$ & - & $1+$ \\
TSGH8301 & A & 2 & $1+$ & $2+$ & $2+$ & $1+$ & \pm & $2+$ & \pm \\
TCC8704 & D2 & 3 & $2+$ & $3+$ & $2+$ & $2+$ & - & $1+$ & \pm \\
\hline
\end{tabular}

Results are expressed by the degree of positive staining: $3+$, strong; $2+$, moderate; $1+$, weak; \pm , faint and -, negative. aJewett staging system. MMPs, matrix metalloproteinases; TIMPs, tissue inhibitors of metalloproteinases; TCC, transitional cell carcinoma.

MA, USA). MGH-U1 and -U1R are sublines of T24, MGH-U3 was established from a grade 1, stage A bladder tumor, and MGH-U4 was established from a stage $\mathrm{O}$ bladder tumor with atypia. The remaining cell lines, TCC8704 and TSGH8301, were courtesy of Dr D.S. Yu (Department of Urology, Tri-Service General Hospital, Taipei, Taiwan, R.O.C.). Cells were incubated at $37^{\circ} \mathrm{C}$ in a $\mathrm{CO}_{2}$ incubator in humidified atmosphere containing $95 \%$ air and $5 \% \mathrm{CO}_{2}$. Table I shows the grade and stage of tumors from which the cell lines were derived. Following Ethic approval and patients' written consent, 30 primary bladder tumor specimens were resected at Chang Gung Memorial Hospital, Taiwan, and studied. Tumor staging was performed based on the 1997 tumor, nodes and metastasis (TNM) classification of bladder cancer, which was agreed upon by the American Joint Committee on Cancer (AJCC) and the Union Internationale Contra Cancer (UICC). Of the 30 resected specimens, 15 were superficial involvements (Ta-T1), 9 had muscle invasion (T2), 4 perivesical invasion (T3) and 2 distant metastases (M1). The histology was 5 grade 1, 14 grade 2 and 11 grade 3 , respectively. Surgical specimens were immersed in plastic containers with optimum cutting temperature compound and stored in a $-70^{\circ} \mathrm{C}$ refrigerator until use.

Chamber slide cultures and immunocytochemistry. Cells $\left(5 \times 10^{4}\right)$ were grown on each well of Lab-Tek ${ }^{\circledR}$ chamber slides (Nunc, Naperville, IL, USA) until confluence was achieved. Immunostaining was carried out at room temperature using the avidin-biotin-peroxidase complex (ABC) method (Vectastain ABC kit, Vector Laboratories, Burlingame, CA, USA), according to the manufacturer's instructions. Briefly, $100 \mu \mathrm{l}$ monoclonal antibodies at a concentration of $5 \mu \mathrm{g} / \mathrm{ml}$, or at the dilution recommended by the manufacturer were added to each well. The slides were incubated for $1 \mathrm{~h}$ following $1 \mathrm{~h}$ of normal horse serum blocking. After washing twice with PBS solution, secondary antibodies (anti-mouse $\operatorname{IgG}$ ) were added and incubated for $1 \mathrm{~h}$. The slides were washed twice with PBS and then $\mathrm{ABC}$ reagent was added and incubated for another $40 \mathrm{~min}$. After washing, the chromogen, 3-amino-9-ethylcarbazol (Aldrich Chemical Co., Inc., Milwaukee, WI, USA), containing $0.02 \%$ hydrogen peroxide, was used to detect the peroxidase conjugation. Gill's hematoxylin solution (Fisher Scientific, Norcross, CA, USA) was used for counterstaining. Monoclonal antibodies (mAbs) used in this study were those against MMP-1 (41-1ES), MMP-2 (42-5D11; Calbiochem, San Diego, CA, USA), MMP-3 (55-2A4), MMP-9, TIMP-1 and TIMP-2 (Oncogene, Cambridge, MA, USA). A negative control (PBS instead of primary antibody, isotype-matched irrelevant mMAbs and normal mouse IgG) and positive controls (antiHLA-A-B,C, W6/32) were included in each test.

Western blot analysis. Cell line extracts were obtained as previously described (2). Blots were incubated overnight with mouse-anti-MMP-2 (Lab Vision, USA) (1 $\mu \mathrm{g} / \mathrm{ml}$ in 3\% BSA) followed by incubation with goat anti-mouse $\mathrm{IgG}$ horseradish peroxidase, developed using the Santa Cruz enhanced chemiluminescence detection system (Santa Cruz Biotechnology, Santa Cruz, CA, USA) and exposed by the Chemi-Smart 3000 (Vilber Lourmat) image system.

Gelatin zymography. Each cell line was grown in culture medium and protein extract was obtained at days 1, 3 and 5 for zymographic analysis. Gelatinolytic zymography was performed as described by Brown et al (3). Briefly, $30 \mu \mathrm{g}$ of protein extract was mixed with non-reducing electrophoresis buffer on a $10 \%$ polyacrylamide gel containing gelatin $(1 \mathrm{mg} / \mathrm{ml})$. After electrophoresis, the gels were incubated in a buffer containing Triton X-100 2.5\%, $0.15 \mathrm{M} \mathrm{NaCl}, 10 \mathrm{mM}$ $\mathrm{CaCl}_{2}, 50 \mathrm{mM}$ Tris-HCl buffer ( $\left.\mathrm{pH} 7.5\right)$ and $0.05 \%$ Coomassie brilliant blue and destained in $7 \%$ acetic acid and $5 \%$ methanol overnight with gentle rocking.

Immunohistochemical staining of bladder tumor tissues. Frozen tissue was cut $(5 \mu \mathrm{m})$ and placed on gelatin-coated slides. The tissue was air-dried and fixed in chilled $\left(4-5^{\circ} \mathrm{C}\right)$ acetone for $10 \mathrm{~min}$. Immunostaining was carried out at room temperature using the ABC method, similar to that described above.

Statistical analysis. The significance of differences was calculated using Chi-square and Fisher's exact probability tests. $\mathrm{P}<0.05$ was considered to be statistically significant.

\section{Results}

Expression of MMPs and TIMPs by TCC cell lines. Immunohistochemical results of MMPs and TIMPs in TCC cell lines are shown in Table I. The cell lines were strongly 


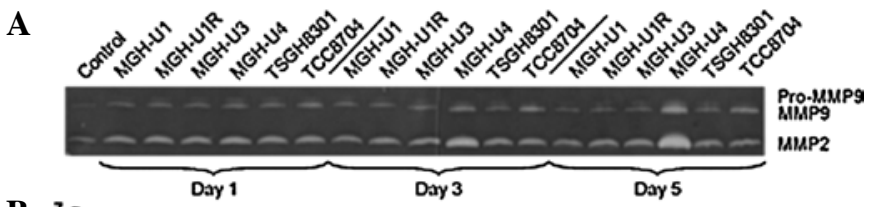

Table III. Expression of MMPs and TIMPs in frozen sections according to tumor grade.

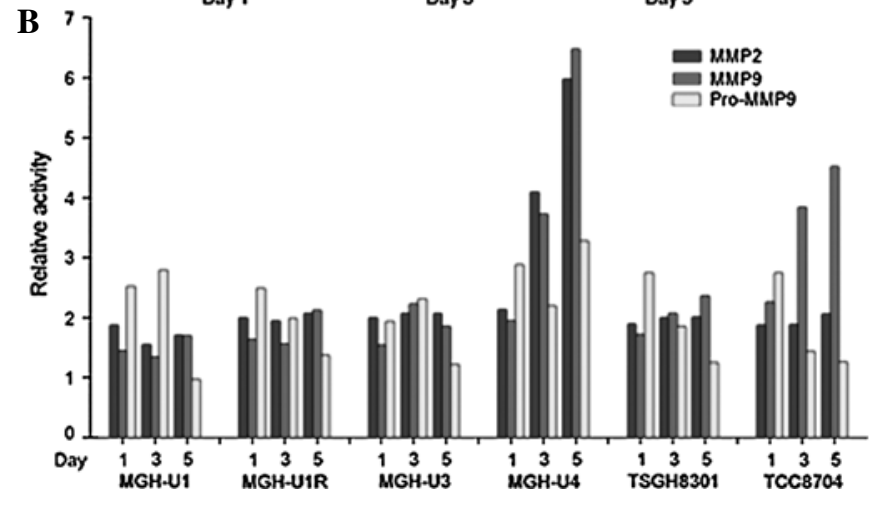

Figure 1. Gelatin zymographic analysis of MMP-2 and -9 in the condition media of six cultured TCC cell lines at days 1, 3 and 5 (A) and quantification of their activities after subtraction of background values (medium control) (B). In (B), the black bar is MMP-2 with a molecular mass of $72 \mathrm{kDa}$, the dark grey bar is MMP-9 with a molecular mass of $84 \mathrm{kDa}$ and the light grey bar is pro-MMP-9 with a molecular mass of $92 \mathrm{kDa}$.

Table II. Expression of MMPs and TIMPs in frozen sections of bladder cancer specimens.

\begin{tabular}{lrc}
\hline & $\mathrm{n}=30$ & Positive (\%) \\
\hline MMP-1 & $30 / 30$ & 100.0 \\
MMP-2 & $19 / 30$ & 63.3 \\
MMP-3 & $2 / 30$ & 6.7 \\
MMP-9a & $21 / 30$ & 70.0 \\
MMP-9b & $15 / 30$ & 50.0 \\
TIMP-1 & $12 / 30$ & 40.0 \\
TIMP-2 & $20 / 30$ & 66.7 \\
\hline
\end{tabular}

Staining scores of $2+$ and above were marked as positive expression.

stained with MMP-1 and weakly stained with MMP-9b. MMP-2 and -3 were moderately stained on MGH-U3, MGH-U4, TSGH8301 and TCC8704, but negatively stained on high-grade TCC MGH-U1 and -U1R. No cell lines expressed TIMP-1 and MMP-9a. Zymographic analysis of the cell lines showed that the level of MMP-2 was found to be higher in MGH-U4 as compared to the other cell lines, and MMP-9 was higher in MGH-U4 and TCC8704 (Fig. 1). Western blotting was used to verify the presence of MMP-2 and -9 in cultured cell lines used in zymography. The expression of MMP and TIMP families was not correlated with the disease status of the original tumors.

Expression of MMPs and TIMPs by bladder carcinoma tissues. The surgical specimens stained positively with MMP-1, with 19 cases being positive for MMP-2 and only 2 specimens being positive for MMP-3 (Table II). The expression of MMP- 2 correlated with grade 3 tumors $(\mathrm{p}=0.036$, Table III). The expression of MMP-9a and MMP-9b correlated

\begin{tabular}{lccc}
\hline $\begin{array}{l}\text { MMP or TIMP } \\
\text { expression }\end{array}$ & $\begin{array}{c}\text { Grade I and II } \\
(\mathrm{n}=19)(\%)\end{array}$ & $\begin{array}{c}\text { Grade III } \\
(\mathrm{n}=11)(\%)\end{array}$ & p-value \\
\hline MMP-1 & $19(100)$ & $11(100)$ & \\
MMP-2 & $9(47.4)$ & $10(90.9)$ & 0.036 \\
MMP-3 & 0 & $2(18.2)$ & 0.068 \\
MMP-9a & $14(73.7)$ & $7(63.6)$ & 0.463 \\
MMP-9b & $9(47.4)$ & $6(54.5)$ & 0.699 \\
TIMP-1 & $5(26.3)$ & $7(63.6)$ & 0.058 \\
TIMP-2 & $12(63.2)$ & $8(72.7)$ & 0.453 \\
\hline
\end{tabular}

Table IV. Expression of MMPs and TIMPs in frozen sections according to tumor stage.

\begin{tabular}{lccc}
\hline $\begin{array}{l}\text { MMP or TIMP } \\
\text { expression }\end{array}$ & $\begin{array}{c}\text { Stage Ta-T1 } \\
(\mathrm{n}=15)(\%)\end{array}$ & $\begin{array}{c}\text { Stage T2, T3 } \\
\text { and M1 } \\
(\mathrm{n}=15)(\%)\end{array}$ & p-value \\
\hline MMP-1 & $15(100)$ & $15(100)$ & 1 \\
MMP-2 & $9(60.0)$ & $10(66.7)$ & 0.686 \\
MMP-3 & $2(13.3)$ & 0 & 0.142 \\
MMP-9a & $7(46.7)$ & $14(93.3)$ & 0.012 \\
MMP-9b & $4(26.7)$ & $11(73.3)$ & 0.023 \\
TIMP-1 & $6(40.0)$ & $6(40.0)$ & 1 \\
TIMP-2 & $11(73.3)$ & $8(53.3)$ & 0.430 \\
\hline
\end{tabular}

with stage $\mathrm{T} 2 / \mathrm{T} 3 / \mathrm{M} 1$ tumors ( $\mathrm{p}=0.012$ and 0.023 , respectively, Table IV). The expression of MMP-1, MMP-3, TIMP-1 and TIMP-2 did not correlate with either tumor stage or tumor grade.

\section{Discussion}

Bladder cancer is the fourth most common malignant neoplasm in men and the eighth most common in women among Americans. Bladder cancer can be classified as superficial and invasive. Additionally, the majority of the bladder tumors are primarily superficial, but $70 \%$ of them will recur and $20-30 \%$ result in progression and metastasis (4). The aims of management of bladder cancer are two-fold: i) to detect relapse of the disease prior to the development of overt symptoms, such as gross hematuria or pain, and ii) to identify tumors that potentially indicate early recurrence, invasion and dissemination. Several studies have attempted to define the most predictable markers for recurrence and metastasis (5). The most common prognostic factors are staging and grading according to pathological characteristics. However, $36 \%$ of patients with urothelial cancer lack these characteristics. Previously, cytological analysis was used for transitional cell carcinomas. However, new tumor markers and molecules are currently under investigation (5). Notably, when considering treatment modalities for patients with transitional cell carcinoma of the bladder it is crucial to identify tumors that 
are likely to progress to invasive disease. Metastasis begins with the growth of tumor cells and invasion into the stroma surrounding the primary neoplasm. Degradation of the extracellular matrix and basement membrane are believed to be associated with tumor invasion and metastasis $(1,6,7)$. The principal intrinsic components of the basement membrane are laminine and type IV collagen. Type IV collagen differs from the interstitial collagens (type I, II and III) in that it is localized exclusively in the basement membrane. Various types of matrix degradative enzymes or proteases are expressed and/ or secreted by tumor cells. These include the metalloproteinases (8), serine proteinases (9) and lysosomal proteases $(10,11)$. MMPs are a family of peptidae enzymes involved in remodeling extracellular components (12), including collagen, gelatin, fibronectin, laminin and proteoglycan. MMPs are complex regulators of multiple cell functions. Different cell types express various MMPs in cancer progression and metastasis (13). Kitagawa et al (14) noted that MMP-2 or gelatinase A is able to degrade type IV collagen and plays a role in tumor angiogenesis and metastasis. Numerous studies focused on the roles of MMPs in tumor invasion and metastasis $(15,16)$. The roles of MMPs in angiogenesis are dual and complex and the increased expression of MMPs has been reported in various human malignant tumors (17-20). MMP-2 and -9 are critical for the angiogenic switch when the tumors become vascularized (21). MMPs were secreted in urine or serum by the tumors, and a zymographic analysis revealed that the urinary levels of MMP-9 and activated MMP-2 were higher in invasive bladder cancers than in superficial ones $(22,23)$. However, in this study, the zymographic analysis of the cell lines did not yield similar results.

TIMP was originally identified as a mammalian collagenase inhibitor and is a glycoprotein with a molecular mass of approximately $28 \mathrm{kDa}(24,25)$. TIMP-2 is a non-glycosylated protein with a molecular mass of approximately $21 \mathrm{kDa}$ and a structure homologous to TIMP. TIMPs are secreted by many types of cells, including tumor cells, and inhibit the activities of various MMPs. In addition, these inhibitors suppress tumor cell invasion in vitro $(26,27)$ and experimental metastasis in vivo $(3,27,28)$. The hypothesis that TIMPs act as tumor suppressor genes due to their anti-metalloproteinase activity and their protective role on the extracellular matrix has been noted $(29,30)$. On the other hand, it was proposed that the simultaneous cellular expression of MMPs and TIMPs in patients with breast cancer be determined as a predictor of clinical outcome (31). No significance of the MMP/TIMP ratio in predicting invasiveness was found in this study.

Our immunohistochemical results showed that MMP-9 was associated with the invasiveness of TCC and MMP-2 was associated with the grade of TCC. A discrepancy was found between the results of frozen sections and chamber slide cell stain, in which tumors of higher grade were stained negatively with MMP-2. Possible causes for the discrepancy in the results may be that the number of cell lines used were insufficent to reflect patient tumors. Additionally, cell lines able to grow in vitro may be highly selective subpopulations of the original tumors. The three-dimensional structure noted in in vivo tumors, as well as the interaction between tumors and stromal cells may be required for MMP-2 expression. Another explanation is that these cell lines were maintained in vitro for more than 2 years and loss of MMP-2 expression occurred following such a long-term in vitro culture. Early stage immunohistochemistry is therefore required to verify this assumption. MMP-9 has been reported to be overexpressed in invasive bladder cancers (23). However, MGH-U1 and -U1R cells were stained negatively with MMP-9. The explanation in MMP-2 may be applied to that of MMP-9. In a given tumor microenvironment, the interaction among tumor cells in situ and tumor-associated cells, such as neutrophils, macrophages, lymphocytes and endothelial cells, as well as environmental factors (hypoxia and $\mathrm{pH}$ ), cytokines and growth factors released by these cells may be required for TCC expression of selective MMPs and TIMPs. The selective expression of these molecules then regulates tumor progression and angiogenesis. Therefore, the immunohistochemical results of the expression of MMPs and TIMPs in TCC tumor specimens may have greater clinical relevance than those obtained with the limited number of TCC cell lines in this study.

CD44 is a cell surface transmembrane glycoprotein that participates in cell motility and adherence of cells to extracellular matrix (ECM). CD44 also modulates the secretion and activation of MMP-2 (32). The collaboration between MMPs and CD44 at the cell surface may be essential in mediating degradation of the ECM to facilitate cell migration (33). In our previous study, a clear correlation of weak or negative staining of CD44v5 and surgical specimen tumor grades and stages was observed (2). The combined weight of the previous (2) and present results indicate that loss of CD44v5 expression may be induced by MMP-2 expressed by high-grade urothelial carcinomas, exhibiting a more invasive phenotype.

In conclusion, immunohistochemical testing of MMP and TIMP expression on 30 TCC surgical specimens revealed that the overexpression of MMP-2 was correlated with tumor grade, while that of MMP-9 was correlated with tumor stage. However, the expression of TIMP-1 or TIMP-2 did not significantly correlate with any of the disease states analyzed, although there was a tendency for TIMP-2 to correlate with tumor grade.

\section{References}

1. Liotta LA: Tumor invasion and metastasis: role of the extracellular matrix. Cancer Res 46: 1-7, 1986.

2. Chuang CK and Liao SK: Differential expression of CD44 variant isoforms by cell lines and tissue specimens of transitional cell carcinoma. Anticancer Res 23: 4635-4640, 2003.

3. Brown PD, Levy AT, Margulies IMK, Liotta LA and Stetler-Stevenson WG: Independent expression and cellular processing of Mr 72,000 type IV collagenase and interstitial collagenase in human tumorigenic cell lines. Cancer Res 50: 6184-6191, 1990

4. Fleshner NE, Herr HW, Stewart AK, Murphy GP, Mettline C and Menck HR: The national cancer data base report on bladder carcinoma. Cancer 77: 1505-1513, 1996.

5. Chuang CK and Liao SK: Evaluation of CA19-9 as a tumor marker in urothelial malignancy. Scand J Urol Nephrol 38: 359-365, 2004.

6 Liotta LA, Tryggvason K, Garbisa S, Hart I, Foltz CM and Shafie S: Metastatic potential correlates with enzymatic degradation of basement membrane collagen. Nature 284: 67-68, 1980.

7. Koivunen E, Ristimaki A, Itkonen O, Osman S, Vuento M and Stenman UH: Tumor-associated trypsin participates in cancer cell mediated defradation of extracellular matrix. Cancer Res 51: 2107-2112, 1991. 
8. Van Wart HE and Birkedal-Hansen H: The cysteine switch: a principle of regulation of metalloproteinase activity with potential applicability to the entire matrix metalloproteinase gene family. Proc Natl Acad Sci USA 87: 5578-5582, 1990.

9. Mignatti P and Rifkin DB: Biology and biochemistry of proteinases in tumor invasion. Physiol Rev 73: 161-195, 1993.

10. Liu BCS and Liotta LA: Biochemistry of bladder cancer invasion and metastasis: clinical implications. Urol Clin North Am 19 621-627, 1992

11. Redwood SM, Liu BCS, Weiss RE, Hodge DE and Droller MJ: Abrogation of the invasion of human bladder tumor cells by using protease inhibitor(s). Cancer 69: 1212-1219, 1992.

12. Liotta LA and Stetler-Stevenson WG: Tumor invasion and metastasis: an imbalance of positive and negative regulation. Cancer Res 51: 5054-5059, 1991.

13. Agnes N, Maud J and Erik M: Matrix metalloproteinases at cancer tumor-host interface. Semin Cell Dev Biol 19: 52-60, 2008.

14. Kitagawa Y, Kunimi K, Ito H, et al: Expression and tissue localization of membrane-types 1, 2 and 3 matrix metalloproteinases in human urothelial carcinomas. J Urol 160: 1540-1545, 1998.

15. Nakajima M, Welch DR, Belloni PN and Nicolson GL: Degradation and basement membrane type IV collagen and lung subendotherial matrix by rat mammary adenocarcinoma cell clones of differing metastatic potentials. Cancer Res 47: 4869-4876, 1987.

16. Nakajima M, Welch DR, Wynn DM, Tsuruo T and Nicolson GL: Serum and plasma Mr 92,000 progelatinase levels correlate with spontaneous metastasis of rat $13762 \mathrm{NF}$ mammary adenocarcinoma. Cancer Res 53: 5802-5807, 1993.

17. Pyke C, Ralfkiaer E, Tryggvason K and Dano K: Messenger RNA for two type IV collagenases is located in stromal cells in human colon cancer. Am J Pathol 142: 359-365, 1993.

18. Stearns ME and Wang M: Type IV collagenase (Mr 72,000) expression in human prostate: benign and malignant tissue. Cancer Res 53: 878-883, 1993

19. Kanayama HO, Yokota KY, Kurokawa Y, Murakami Y, Nishitani $M$ and Kagawa S: Prognostic values of matrix metalloproteinase-2 and tissue inhibitor of metalloproteinase-2 expression in bladder cancer. Cancer 82: 1359-1366, 1998.

20. Grignon DJ, Sakr W, Toth M, et al: High levels of tissue inhibitor of metalloproteinase-2 (TIMP-2) expression are associated with poor outcome in invasive bladder cancer. Cancer Res 56: 1654-1659, 1996.
21. Hanahan D and Folkman J: Patterns and emerging mechanisms of the angiogenic switch during tumorigenesis. Cell 86: 353-364, 1996.

22. Davis B, Waxman J, Wasan $\mathrm{H}$, et al: Levels of matrix metalloproteinase in bladder cancer correlate with tumor grade and invasion. Cancer Res 53: 5365-5369, 1993.

23. Gerhards S, Jung K, Koenig F, et al: Excretion of matrix metalloproteinases 2 and 9 in urine is associated with a high stage and grade of bladder carcinoma. Urology 57: 675-679, 2001.

24. Cawston TE, Galloway WA, Mercer E, Murphy G and Reynolds JJ: Purification of rabbit bone inhibitor for collagenase. Biochem J 195: 159-165, 1981.

25. Docherty AJ, Lyons A, Smith BJ, et al: Sequence of human tissue inhibitor of metalloproteinases and its identity to erythroid-potentiating activity. Nature 318: 66-69, 1985.

26. Mignatti P, Robbins E and Rifkin DB: Tumor invasion through the human amniotic membrane: requirement for a proteinase cascade. Cell 47: 487-498, 1986.

27. Schultz RM, Silberman S, Persky B, Bajkowski AS and Carmichael DF: Inhibition by human recombinant tissue inhibitor of metalloproteinases of human amnion invasion and lung colonization by murine B16-F10 melanoma cells. Cancer Res 48: 5539-5545, 1988.

28. Alvarez OA, Carmichael DF and DeClerck YA: Inhibition of collagenolytic activity and metastasis of tumor cells by a recombinant human tissue inhititor of metalloproteinases. J Natl Cancer Inst 82: 589-595, 1990.

29. Stetler-Stevenson WG, Krutzsch HC and Liotta LA: TIMP2: identification and characterization of a new member of the metalloproteinases inhibitor family. Matrix Supplement 1: 299-306, 1992.

30. Denhardt DT, Khokha R, Yagel S, Overall CM and Parhar RS: Oncogenic consequences of down-regulating TIMP expression in 3T3 cells with antisense RNA. Matrix Supplement 1: 281-285, 1992.

31. Baker EA, Stephenson TJ, Reed MW and Brown NJ: Expression of proteinases and inhibitors in human breast cancer progression and survival. Mol Pathol 55: 300-304, 2002.

32. Takahashi K, Eto H and Tanabe KK: Involvement of CD44 in matrix metalloproteinase-2 regulation in human melanoma cells. Int J Cancer 29: 387-395, 1999.

33. Isacke $\mathrm{CM}$ and Yarwood $\mathrm{H}$ : Molecules in focus. The hyaluronan receptor, CD44. Int J Biochem B 34: 718-721, 2002. 